\title{
Adaptive Traffic Light Control using Google Maps API at Multiple Road Intersections
}

\author{
Adhish P, Abhijith K, Sree Ram M
}

\begin{abstract}
In today's urbanized world one of the major and regular crisis we face is Traffic Congestion, this crisis is caused by an increase in the usage of private vehicles adjoint with the availability in the land resources present. This major crisis has increased the attention of the research and development community and has led to the development of various Intelligent Traffic Management System(ITS).In this paper we present a solution by using Google Maps API which is a crowdsourced data used for detecting trafficked areas and these values are transferred to a private cloud storage where a traffic congestion calculation algorithm is present and this algorithm is used to categorize the status of congestion for a particular intersection.
\end{abstract}

Keywords: Traffic Light control, Google Maps API, Traffic Congestion Management.

\section{INTRODUCTION}

Urbanized cities all over the world face a similar problem of Traffic congestion as well as productive time and nonrenewable resources such as petroleum and diesel. It is a condition caused by the increasing number of vehicles on the road resulting in congestion, slower speed, blockage of roads, longer route time and the loss of precious time. It is a serious issue faced by every city these days. Traffic jam leads to sheer waste of productive time. The congestion mostly occurs during peak hours on the main roads when people commute to work or on their way back home. The Industrial development in the recent years has only increased the problem of traffic congestion by increasing number of transport vehicles. On an average, a person spends around 30 mins to 2 hours in their day driving. Most of this time is spent in traffic jams. These issues have motivated increased attention from the research community and led to the development of a variety of vehicular traffic management (VTM) strategies. This paper presents a strategy for an adaptive traffic light phase and duration optimization algorithm that factors in the road conditions of multiple intersections using Google Maps APIs. In this we use Google maps APIs which is a crowd sourced data which can be efficiently used to analyze the traffic in a particular intersection. Using the data that was analyzed we can control

Revised Manuscript Received on December 30, 2019.

* Correspondence Author

Adhish.P*, Student, Dept of Computer Science \& Engineering SRM Institute of Science \& Technology, Ramapuram.

Abhijith.K, Student, Dept of Computer Science \& Engineering SRM Institute of Science \& Technology, Ramapuram.

Sreeram.M, Student, Dept of Computer Science \& Engineering SRM Institute of Science \& Technology, Ramapuram.

(C) The Authors. Published by Blue Eyes Intelligence Engineering and Sciences Publication (BEIESP). This is an open access article under the CC BY-NC-ND license (http://creativecommons.org/licenses/by-nc-nd/4.0/) the traffic light signal adjuster at an intersection. The collected API codes are stored in a private cloud storage where the process of duration optimization takes place using the ATOM algorithm. Then the resultant data stored in the respective controlled rooms are forwarded to the road side unit through the control officer who holds the resultant data. Then the road side unit acts according to the resultant data and manipulates the traffic signal present. Hence, using our proposed system traffic can be cleared simultaneously and efficiently by managing the traffic lights. This also contains various features like clearly traffic instantly for emergency vehicles like ambulances and fire engines through which numerous lives are saved.

\section{LITERATURE SURVEY}

Urban traffic congestion propagation and bottleneck identification done by Long J, Gao Z, Ren H and Lian A published on June 2015. It estimates the average journey velocity (AJV) of both link and network to identify network congestion bottlenecks. It identifies network bottlenecks and improves network traffic condition and prevents traffic congestion.

A methodology for road traffic resilience analysis and review of related concepts by Simeon C. Calvert published on August 2017. It is able to detect poorly and weak resilient locations by analyzing the relative resilient value of individual road sections. The total delay experience on a road section gives an overall indication of the negative effect of congestion on a road section. Evaluation of fluctuating speed and lateral movement of vehicles by Sai Chand and Vinayak Dixit published on January 2016. The proposed method analyses and takes account of the heterogeneity of the speed of the vehicles. At a higher frequency, the proposed method can characterize traffic conditions. Intelligent traffic light control done by Marco Wiering, Jelle van Veenen, Jilles Vreeken, Arne Koopman was published on July 9, 2004. In this paper they have proposed a method to efficiently clear traffic using an algorithm known as adaptive optimization algorithm based upon reinforcement learning. Reinforcement learning is the process of a module interact with its environment and learn from its obtained feedback. They implemented a traffic light simulator in Green Light District which experiments with different infrastructure. Their experimental results indicate adaptive traffic light controllers outperform other fixed controllers. Also, here they use prediction techniques, Evolutionary algorithms, Dynamic programming and markov decision problems. An Adaptive Traffic light control scheme and its implementation in WSN based ITS done by Binbin Zhou, Jiannong Cao, Jingjing Li was published on September 5,2013. 
In this paper they have proposed a method known as Wireless Sensor Network (WSN). They WSN are sensors dedicated for the purpose of monitoring and recording physical conditions and organize the collected data. Here an adaptive traffic light control scheme had been proposed,

where sequence of lights is adjusted dynamically with accordance to the detected real time traffic. The simulation results prove that the proposed scheme can achieve much higher performance, in terms of throughput and average waiting time.

Road Traffic Congestion in the developing world done by Vipin Jain, Ashlesh Sharma, Lakshminarayanan Subramanian was published on March 2012. In this paper they have proposed a simple automated image processing mechanism for detecting the congestion levels in road traffic by processing CCTV camera image feeds. Their algorithm is designed particularly for noisy roads with poor image quality. Based on this image processing they present a local de-congestion protocol that coordinates traffic signal behavior within a small area and can locally prevent congestion collapse sustaining variant traffic bursts.

Cloud computing based adaptive traffic light control work done by P. Jaworkski Published on December 2014.The system uses Intelligent Transportation System (ITS) cloud to host collection of software service that form cloud-based traffic management system. The system does not analyze traffic in real time. The collection of traffic information through sensors cost much and difficult to maintain. Design and implementation of intersection algorithms, ITS-Cloud platform, the Cloud based Traffic Management system and the traffic simulator.

Congestion Adaptive Traffic Light Control and Notification Architecture Using Google Maps APIs work done by Sumit Mishra and Ankit Gupta published on 14 December 2018.This system informs the drivers about the upcoming traffic light signal on their route. It uses crowd sourced traffic congestion data from google. The congestion value calculated is in respect to an hour and, therefore, provides good insight into the temporal congestion values.

\section{TRAFFIC CONGESTION CALCULATION ALGORITHM}

In this module a web application is created which will run the congestion calculating algorithm and display the results. The results of the congestion calculating algorithm will be monitored in the control room by an officer.

The traffic can be altered depending upon the status of the result directly from the control room by using a Wi-Fi panel. By using the Wi-Fi panel, the road side unit (RSU) can be contacted and the traffic light present at that intersection can be changed. The algorithm takes weight factor of each road as input, which is the number of roads linked to the lane. The weight factor is multiplied with the difference of initially grabbed estimated time to travel and general time to travel for each road. The submission is used to give congestion status. $\mathrm{CDV}=\sum[($ Weight factor of the lane 'N') * (Difference in the estimated time for the lane ' $n$ ')]

Where CDV is the congestion delay value, ' $N$ ' is the total number of lanes connected at an intersection and ' $n$ ' is lane number.

The congestion status is determined as follows:

No Congestion:
Congestion delay value $\leq$ (Last week maximum hourly congestion $+2 \times$ Last week minimum hourly congestion)/3 Medium Congestion:

(Last week maximum hourly congestion $+2 \times$ Last week minimum hourly congestion $) / 3<$ Congestion Value $\leq(2 \times$ Last week maximum hourly congestion + Last week minimum hourly congestion)/3

High Congestion:

$(2 \times$ Last week maximum hourly congestion + Last week minimum hourly congestion) $/ 3<$ Congestion Value

\section{ARCHITECTURE DIAGRAM}

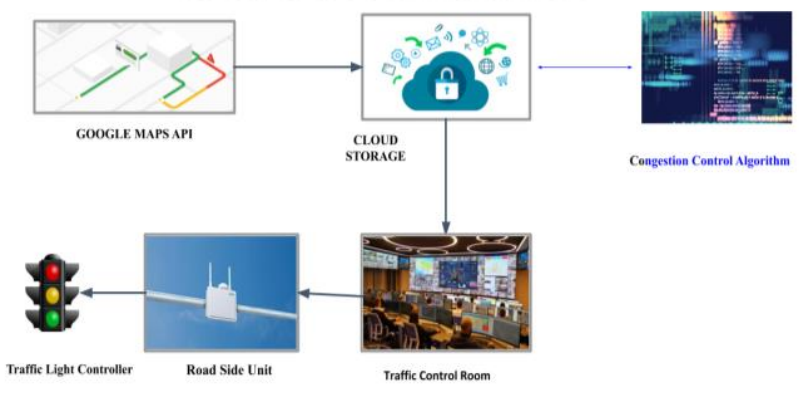

In this project we use google maps API to collect traffic data, the main reason for using these API codes are for getting the instantaneous traffic data into the cloud. The collected data is stored in cloud storage and the Congestion control algorithm is used in a web application in which the level of congestion for the main and its neighboring intersections are calculated. After that the overall congestion value is compared and the congestion status is determined. The result is then displayed in the Traffic control room. Then according to the level of traffic the authorized person can determine the suitable traffic light cycle time, which is already present in the local server of a traffic control room. The traffic lights are changed by contacting the Road Side Unit through a Wi-Fi module.

\section{SYSTEM METHODOLOGY}

\section{A. Collecting Date From API}

The traffic status of a particular location is collected and stored in a cloud-based server. There are several APIs available in the market which provides live traffic details by using sensed data of GPS and many other sensors of our mobile device and are uploaded to the central server where analysis and calculation are done. These processed data are made available to any client device or application in the form of APIs. In this project we use Google Maps API, which is a crowd source and widely used application for monitoring traffic. Google maps API is a feature offered by google in the form of an application, which provides user the details about the geographic status of their location, details about the surrounding buildings, live location of the user using gps, traffic status of the particular road-navigation etc. For the development of new project and for commercial usage of other entities, google offer subscription to the map's API services. 
There are several API features offered by google like Maps, Routes and places. Each feature uses different types of API.

In this project we use Maps JavaScript API which provides real time traffic information to our maps and provide the congestion delay details for a particular route. Once the API is enabled, it generates an API key which is a unique key that is generated to use our own map features.

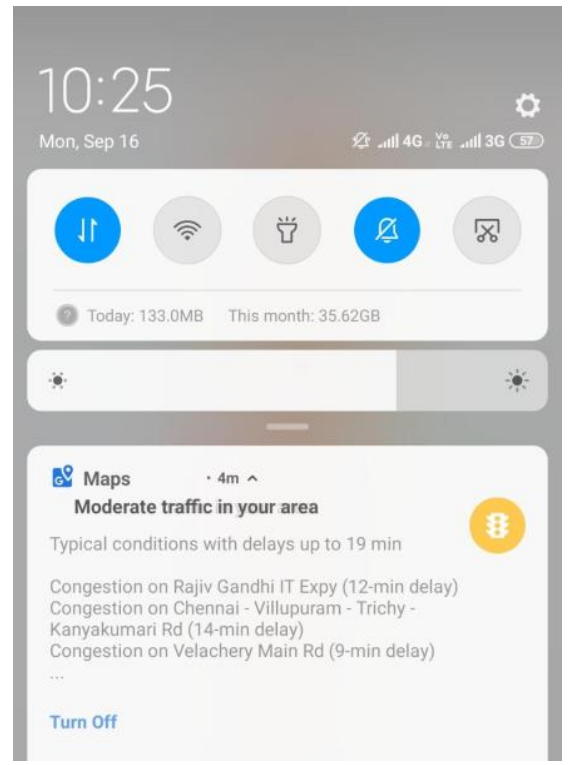

Fig 4.1.1 Delay time notification

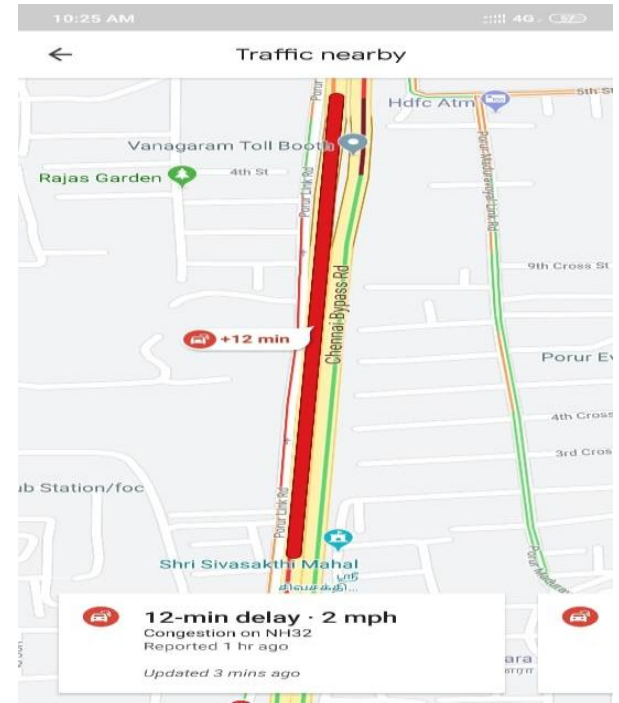

The requested API key is :

$<$ script

async defer

src="https://maps.googleapis.com/maps/api/js?key=YOUR_

API_KEY\&callback=initMap"

type="text/javascript" $></$ script $>$

The collected data is then stored in a cloud-based server for calculation and analysis of the traffic and to execute the congestion control algorithm. There are several cloud platforms available in the market, but google insists to use any one of its own cloud platforms to store the data for project related purposes. The cloud platforms afford by google are GCP console and gsutil cloud storages.
Fig 4.1.2 Real time traffic from Google

\section{B. Traffic Congestion Calculation at Multiple Road Intersection}

If a traffic congestion is calculated with respect to that of the nearby intersections more accuracy of congestion can be derived and hence more time is saved. In this project we consider the neighboring right and left intersections and we are calculating the congestion result. In a single intersection calculation, there is a chance that the traffic in the present intersection can clog along with traffic in the upcoming intersection. This can be avoided by using congestion calculation at multiple intersection. For this calculation first the congestion value for each lane of each intersection is calculated. Then the congestion status of each lane is determined using the congestion value and last week's minimum and maximum hourly congestion. Using the congestion status of each lane the overall congestion status for each intersection is determined. Then by using the congestion status of each intersection, the status of congestion at the targeted intersection is determined.

\section{Changing Traffic Light Signal from Control Room}

The result which is the status of the traffic data is now available in the control room. According to the status of the traffic the traffic signal cycle time can be chosen from the list of cycle times available in the Traffic signal cycle time data available in the control room. The signal is changed via a WIFI module which contacts the Road Side Unit to change the current cycle time to the instruction passed from the control room.

\section{MISC ALGORITHM}

The calculation of congestion status for multiple intersection is as follows:

STEP 1: At first the real time congestion value for each lane is determined by,

$\mathrm{CDV}=\sum[($ Weight factor of the lane 'N') * (Difference in the estimated time for the lane ' $n$ ')]

Where CDV is the congestion delay value, ' $\mathrm{N}$ ' is the total number of lanes connected at an intersection and ' $n$ ' is lane number.

STEP 2: Then the congestion status of each lane is determined by,

No Congestion:

$\mathrm{CDV} \leq$ (Last week maximum hourly congestion $+2 \times$ Last week minimum hourly congestion)/3

Medium Congestion:

(Last week maximum hourly congestion $+2 \times$ Last week minimum hourly congestion $) / 3<\mathrm{CDV} \leq(2 \times$ Last week maximum hourly congestion + Last week minimum hourly congestion) $/ 3$

High Congestion:

$(2 \times$ Last week maximum hourly congestion + Last week minimum hourly congestion) $/ 3<\mathrm{CDV}$

STEP 3: The overall congestion status is determined separately for each intersection by considering the status of each separate paths. 
STEP 4: The final congestion status for multiple intersections is calculated by considering all the possible intersection surrounding the center intersection where the status is being analyzed.

Let's consider the overall congestion status at each intersection as $\mathrm{i} 1, \mathrm{i} 2, \mathrm{i} 3 . . . \mathrm{iN}$ respectively and let the center intersection be Ic.

No congestion: if (Ic status) < (i1,i2,i3...in status)

Medium congestion: if (Ic status) $=(\mathrm{i} 1, \mathrm{i} 2, \mathrm{i} 3$...in status)

High Congestion: if (Ic status) $>(\mathrm{i} 1, \mathrm{i} 2, \mathrm{i} 3$...in status $)$

\section{DEMONSTRATION USING ANYLOGIC}

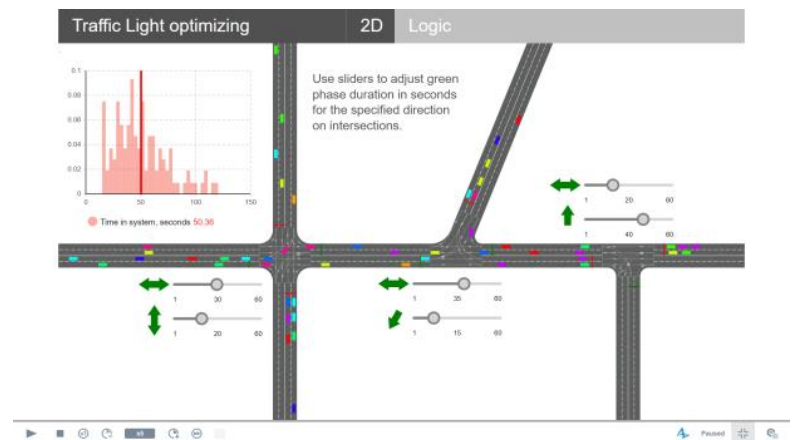

Fig 7.1 MISC Algorithm Simulation

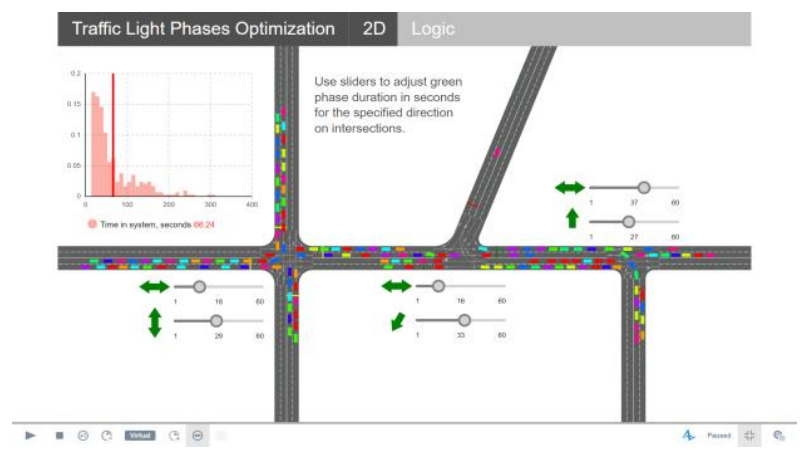

Fig 7.2 SISC Algorithm Simulation

Anylogic software is used for the simulation of the proposed model. In this model a multiple intersection roads are created for the simulation of the traffic in multiple intersection status calculation algorithm as shown in the Fig 7.1.The timing is calculated in seconds and the timing is adjusted through the sliders provided in the model according to the status calculated by MISC algorithm .It has been proved that the MISC algorithm is more effective than the SISC algorithm.

\section{RESULT}

The following graph 8.1 and graph 8.2 displays the congestion value along with real world time in seconds for calculating the mean clearance time of the traffic. To calculate congestion value a sample dataset which contains latitude and longitude details of several locations with delay time has been used. It can be seen that the clearance time in MISC is faster than that of SISC algorithm. The clearance time of MISC is 50.36 whereas SISC clearance time is 73.Hence we conclude that Multiple Intersection Status calculation Algorithm is more efficient than SISC algorithm.

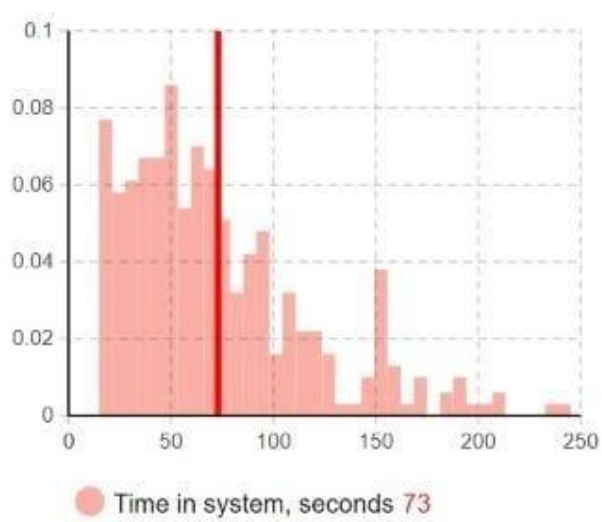

Graph 8.1 SISC Algorithm

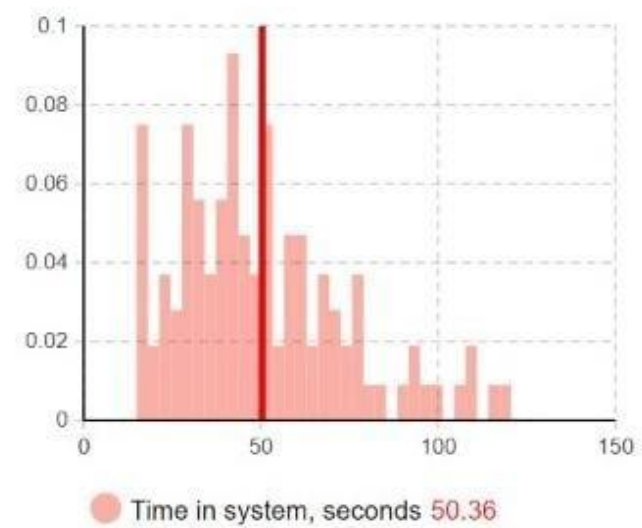

Graph 8.2 MISC Algorithm

\section{CONCLUSION}

In this paper we present the Multiple Road intersection status calculating algorithm. In this algorithm we estimate the volume of incoming traffic and also check the status of congestion for each intersection. The algorithm then sends the congestion status to the local transport authority. Who can then decide the necessary traffic signal cycle time required to control the traffic and reduce the congestion. This methodology is based on analyzing the data collected from the crowdsource APIs like Google API using both real time and the past traffic data to predict the congestion status in the near future. By calculating the status considering multiple intersection more accuracy in the status is achieved and also the clogging of vehicles in the upcoming intersection is avoided. By this way the waiting time of vehicles and accidents caused by traffic congestion is avoided. This system is infrastructure free as there is no need to construct any sensors or much of any road side units to collect the traffic details since we use the google maps API.

This whole system can be very helpful to transport engineers and traffic authority personnel to make important reports using the dataset collected in the database for getting the accurate location of log of vehicles, to make evacuation plan during emergencies, to choose an alternative path during road construction and related activities, to detect the need for optimizing waiting time and much more. 


\section{REFERENCES}

1. Detian Zhang, Yuan Liu, AN Liu, Xudong Mao, and Qing Li, efficient path processing by cloud-based mapping services, IEEE, published on 11 July 2014.

2. MD.Al Amin, MD. Rofi Uddin supervised by Mrs. Sadia Hamid Kazi, Traffic monitoring using GPS data.

3. Divya Jayakumar Nair, Flavien Gillies, Sai Chand, Neeraj Saxena, Vinayak Dixit, multi city urban traffic using crowd sourced data, PLOS ONE, published on 12 March 2019.

4. Inam Ullah Khan, Muhammad Umar Khan, Hafiz Muhammad Salman, Syed Bilal Hussain Shah, Traffic light control using traffic density, Journal of American Academic Research (JAAR), volume 5 issue 2, published on June 2017.

5. Sumit Mishra, Devanjan Bhattacharya and Ankit Gupta, Congestion traffic light control using google maps, MDPI, published on 14 December 2018.

6. Marco Wiering, Jelle van Veenen, Jilles Vreeken, Arne Koopman, Intelligent traffic light control, published on 09 July 2004.

7. Binbin Zhou, Jiannong Cao, Jingjing Li, Traffic light control using wireless sensor network (WSN), International Journal on Smart Sensing and Intelligent Systems, volume 6, published on 05 September 2013.

8. Vipin Jain, Ashlesh Sharma, Lakshminarayanan Subramanian, Road traffic congestion in the developing world, published on march 2012.

9. Pawel Jaworski, traffic control using cloud computing, published on September 2013.

10. Sookung Lee, Mohamed Younis, Aiswarya Murali and Meejeong Lee, dynamic vehicular flow optimization, IEEE, published on 21-02-2019.

\section{AUTHORS PROFILE}

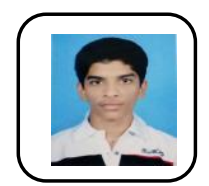

Adhish.P is a pre-final year student in Dept of Computer Science \& Engineering at SRM Institute of Science \& Technology, Ramapuram. He has experience in Mobile Application development and Web designing and development

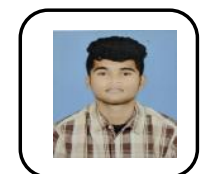

Abhijith.K is a pre-final year student in Dept of Computer Science \& Engineering at SRM Institute of Science \& Technology, Ramapuram. He has experience in Business Intelligence and Database Management Systems

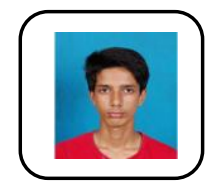

Sreeram.M is a pre-final year student in Dept of Computer Science \& Engineering at SRM Institute of Science \& Technology, Ramapuram. He has experience in Cloud Computing technologies, Web hosting, and Web Designing. 